Supporting Information for:

\title{
RAFT Dispersion Alternating Copolymerization of \\ Styrene with $N$-Phenylmaleimide: Morphology \\ Control and Application as an Aqueous Foam
}

\section{Stabilizer}

\author{
Pengcheng Yang, ${ }^{\dagger}$ Oleksandr O. Mykhaylyk, Elizabeth R. Jones and Steven P. Armes* \\ Department of Chemistry, University of Sheffield,
}

Brook Hill, Sheffield, South Yorkshire, S3 7HF, UK 


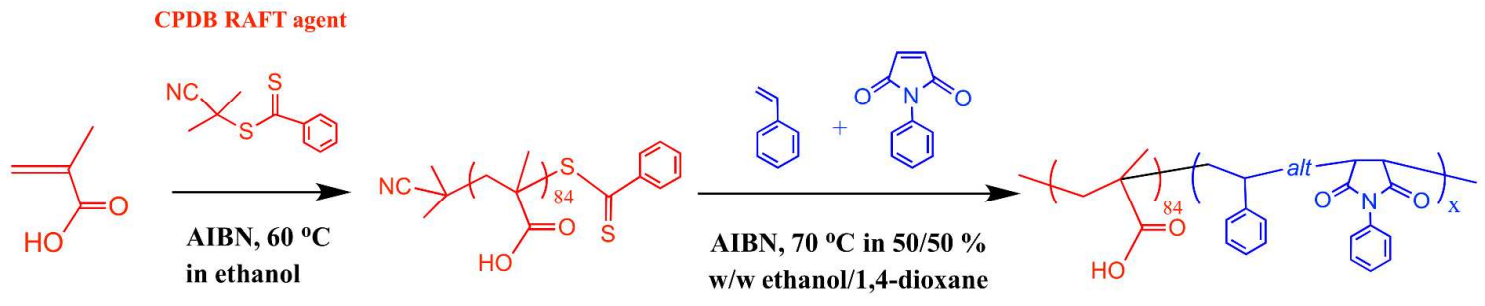

MAA monomer

PMAA84 macro-CTA

PMAA $_{84}-\mathrm{P}(\text { St-alt-NMI) })_{\mathrm{x}}$ diblock copolymer

Scheme S1. RAFT synthesis of poly(methacrylic acid $)_{84}\left(\right.$ PMAA $\left._{84}\right)$ macro-CTA via solution polymerization in ethanol at $60{ }^{\circ} \mathrm{C}$, followed by RAFT dispersion alternating copolymerization of styrene (St) with $N$-phenylmaleimide (NMI) in a 50/50 \% w/w ethanol/1,4-dioxane at $70{ }^{\circ} \mathrm{C}$.

Table S1. Summary of monomer conversions, GPC molecular weight data, DLS particle diameters and TEM morphologies obtained for a series of $\mathrm{PMAA}_{84}-\mathrm{P}(\mathrm{St}-\text { alt-NMI })_{\mathrm{x}}$ diblock copolymers synthesized via RAFT dispersion alternating copolymerization at $70{ }^{\circ} \mathrm{C}$ using AIBN initiator in a 50/50 \% w/w ethanol/1,4-dioxane mixture at $20 \% \mathrm{w} / \mathrm{w}$ solids. $(\mathrm{S}=$ spheres; $\mathrm{W}=$ worms; $\mathrm{L}=$ lamellae). ${ }^{\mathrm{a}}$

\begin{tabular}{|c|c|c|c|c|c|c|c|c|}
\hline \multirow{2}{*}{$\begin{array}{c}\text { Entry } \\
\text { No. }\end{array}$} & \multirow{2}{*}{$\begin{array}{c}\text { Target DP for } \\
\text { core-forming } \\
\text { P(St-alt-NMI) } \\
\text { block }\end{array}$} & \multirow{2}{*}{$\begin{array}{c}\text { Overall } \\
\text { comonomer } \\
\text { conversion } \\
(\%)\end{array}$} & \multirow{2}{*}{$\begin{array}{l}\text { Actual DP for } \\
\text { core-forming } \\
\text { P(St-alt-NMI) } \\
\text { block }^{c}\end{array}$} & \multicolumn{2}{|c|}{ GPC data ${ }^{d}$} & \multicolumn{2}{|c|}{ DLS data $^{e}$} & \multirow{2}{*}{\begin{tabular}{|c|} 
TEM \\
morphology \\
assignment
\end{tabular}} \\
\hline & & & & $M_{n}$ & $\mathbf{M}_{\mathbf{w}} / \mathbf{M}_{\mathbf{n}}$ & $\begin{array}{c}\text { Diameter } \\
\text { (nm) }\end{array}$ & PDI & \\
\hline 1 & 220 & 97 & 214 & 24,700 & 1.33 & 60 & 0.06 & $\mathrm{~S}$ \\
\hline 2 & 420 & 95 & 400 & 34,900 & 1.36 & 220 & 0.28 & W \\
\hline 3 & 700 & 96 & 672 & 43,800 & 1.39 & 450 & 0.38 & $\mathrm{~L}$ \\
\hline
\end{tabular}

${ }^{a}$ Conditions: $[\mathrm{St}] /[\mathrm{NMI}]$ comonomer feed molar ratio $=1.0 ;[$ macro-CTA $] /[\mathrm{AIBN}]$ molar ratio $=5.0$.

${ }^{\mathrm{b}}$ Determined by ${ }^{1} \mathrm{H}$ NMR spectroscopy in $\mathrm{d}_{6}$-DMSO.

${ }^{\mathrm{c}}$ Actual DP of $\mathrm{P}(\mathrm{St}-$ alt $-\mathrm{NMI})=$ target $\mathrm{DP}$ of $\mathrm{P}(\mathrm{St}-$ alt $-\mathrm{NMI}) \times$ overall comonomer conversion

${ }^{\mathrm{d}}$ After exhaustive methylation using excess trimethylsilyl diazomethane and THF GPC (refractive detector, calibrated with polystyrene standards).

${ }^{\mathrm{e}}$ Intensity-average DLS diameters were calculated using cumulants analysis software provided by the instrument manufacturer (Malvern, UK). PDI denotes the DLS polydispersity. 

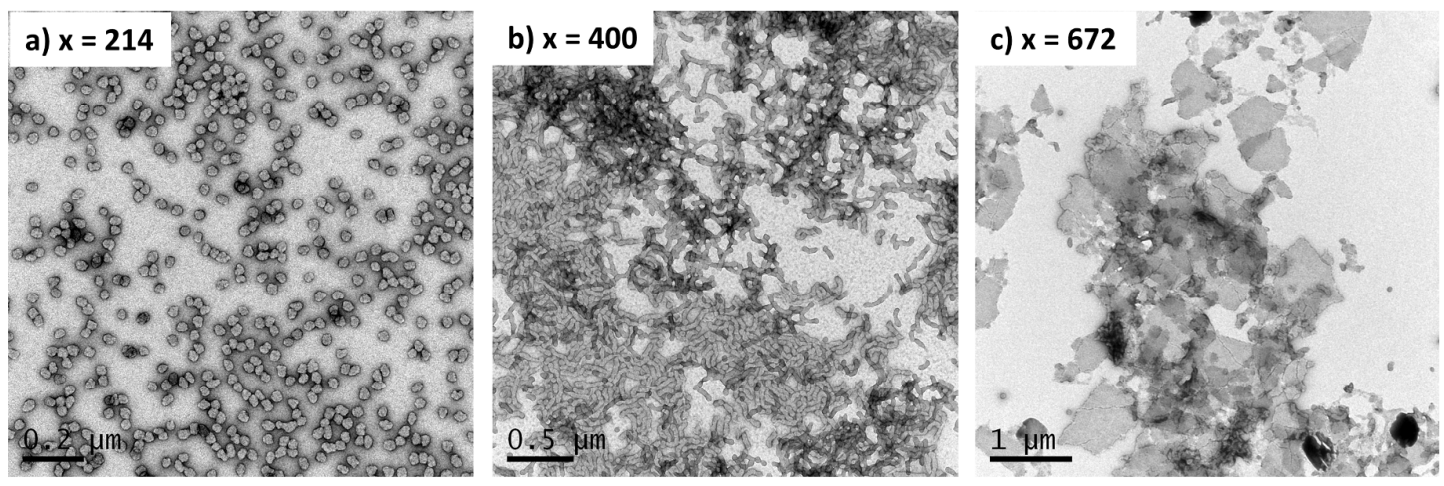

Figure S1. Representative TEM images for a series of $\mathrm{PMAA}_{84}-\mathrm{P}(\mathrm{St}-\text { alt-NMI })_{\mathrm{x}}$ diblock copolymer nano-objects (a) spheres, $\mathrm{x}=214$; (b) worms, $\mathrm{x}=400$; (c) lamellae, $\mathrm{x}=672$ prepared via RAFT dispersion alternating copolymerization at $70{ }^{\circ} \mathrm{C}$ in a $50 / 50 \% \mathrm{w} / \mathrm{w}$ ethanol/1,4-dioxane mixture at $20 \% \mathrm{w} / \mathrm{w}$ solids.

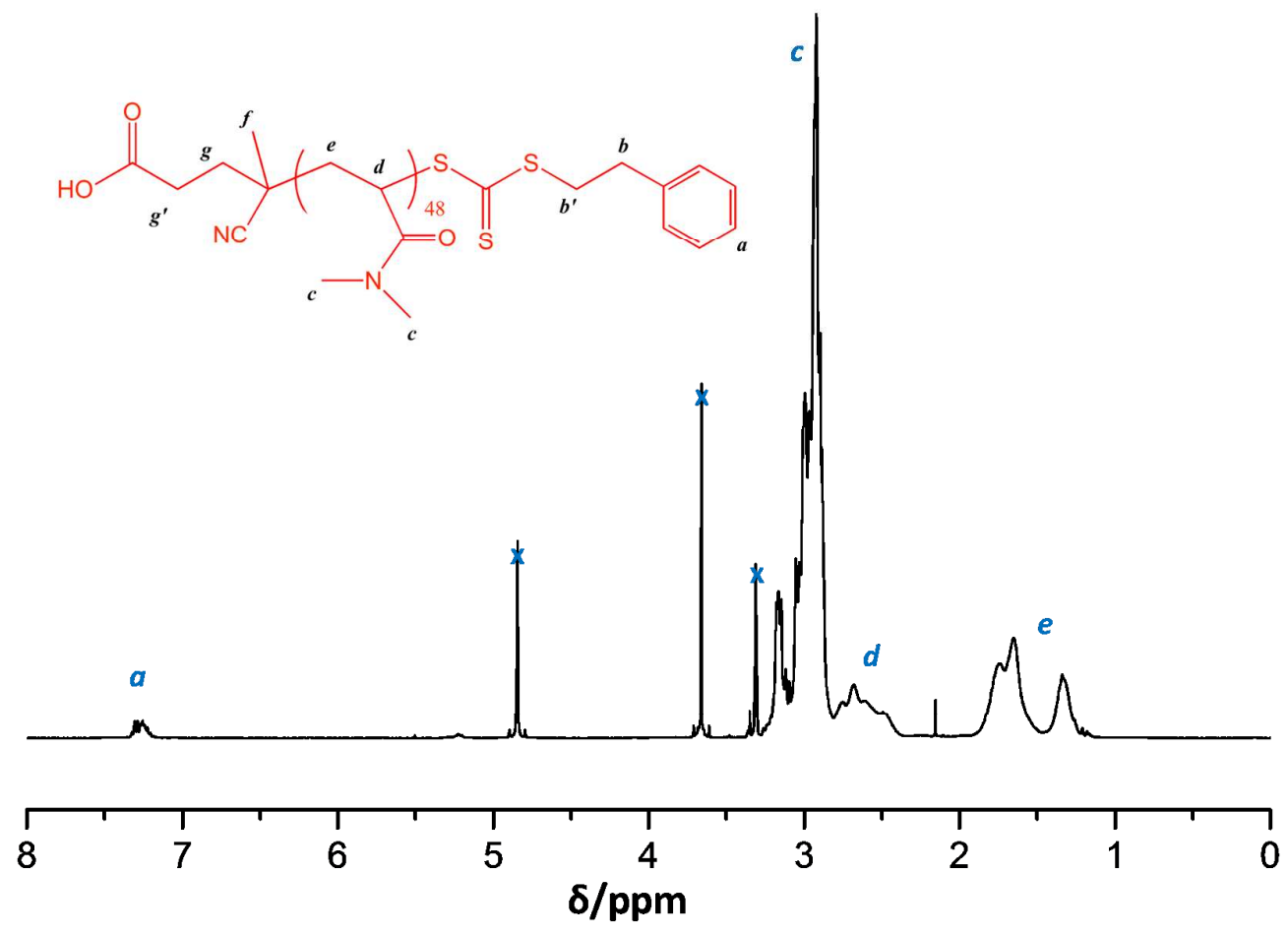

Figure S2. Assigned ${ }^{1} \mathrm{H}$ NMR spectrum ( $\mathrm{d}_{4}$-methanol) for the PDMAC 48 macro-CTA. 


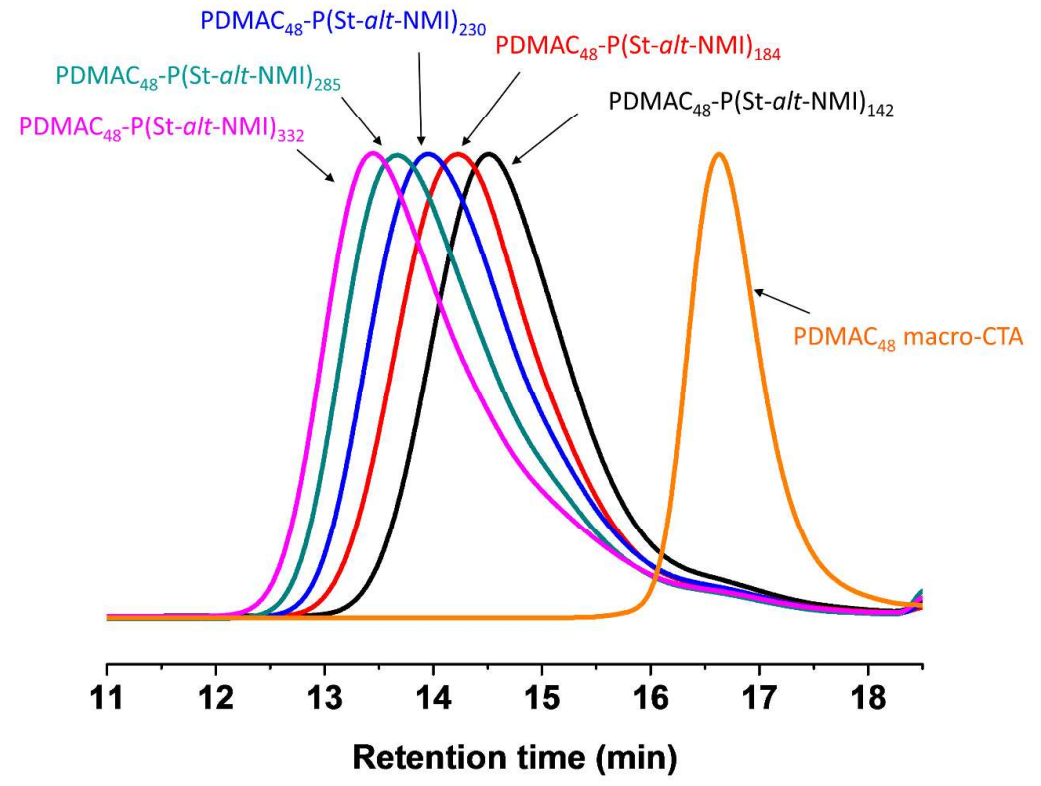

Figure S3. DMF GPC traces obtained for $\mathrm{PDMAC}_{48}$ macro-CTA precursor and various corresponding $\mathrm{PDMAC}_{48} \mathrm{P}(\mathrm{St}-\text { alt-NMI })_{\mathrm{x}}$ diblock copolymers synthesized via RAFT dispersion alternating copolymerization in a 50/50\% w/w ethanol/MEK mixture at $70{ }^{\circ} \mathrm{C}$.

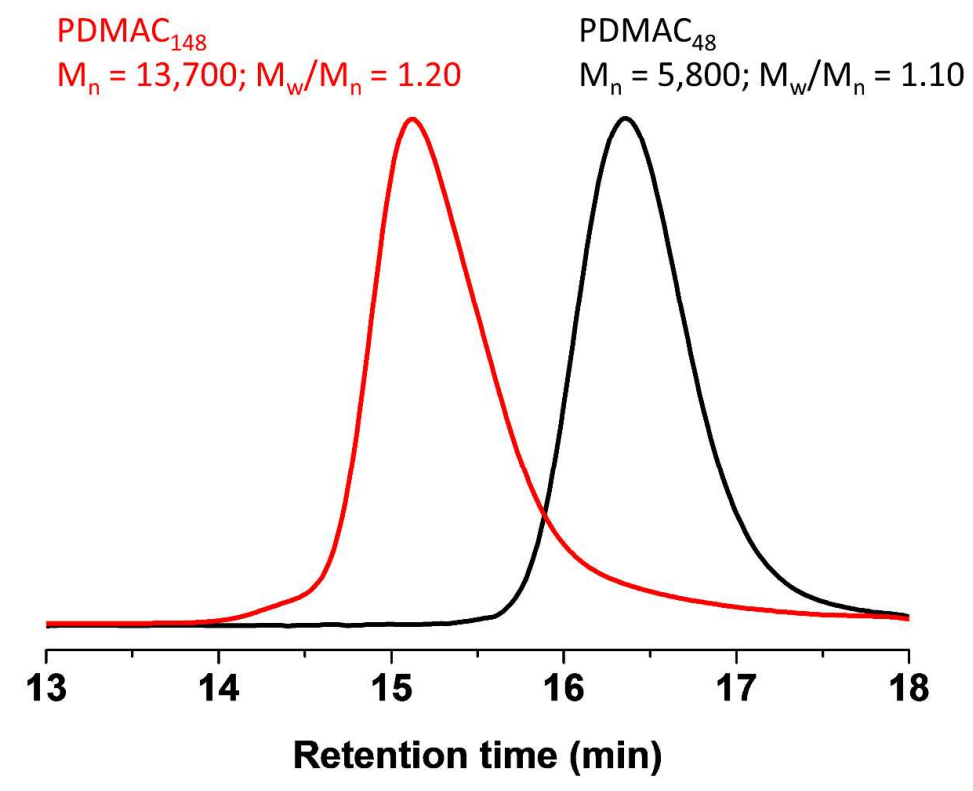

Figure S4. DMF GPC trace (refractive index detector) obtained for the PDMAC 48 macroCTA (black curve). The corresponding self-blocking chain extension experiment (red curve) using 100 units of DMAC confirms a relatively high blocking efficiency for this macro-CTA. 


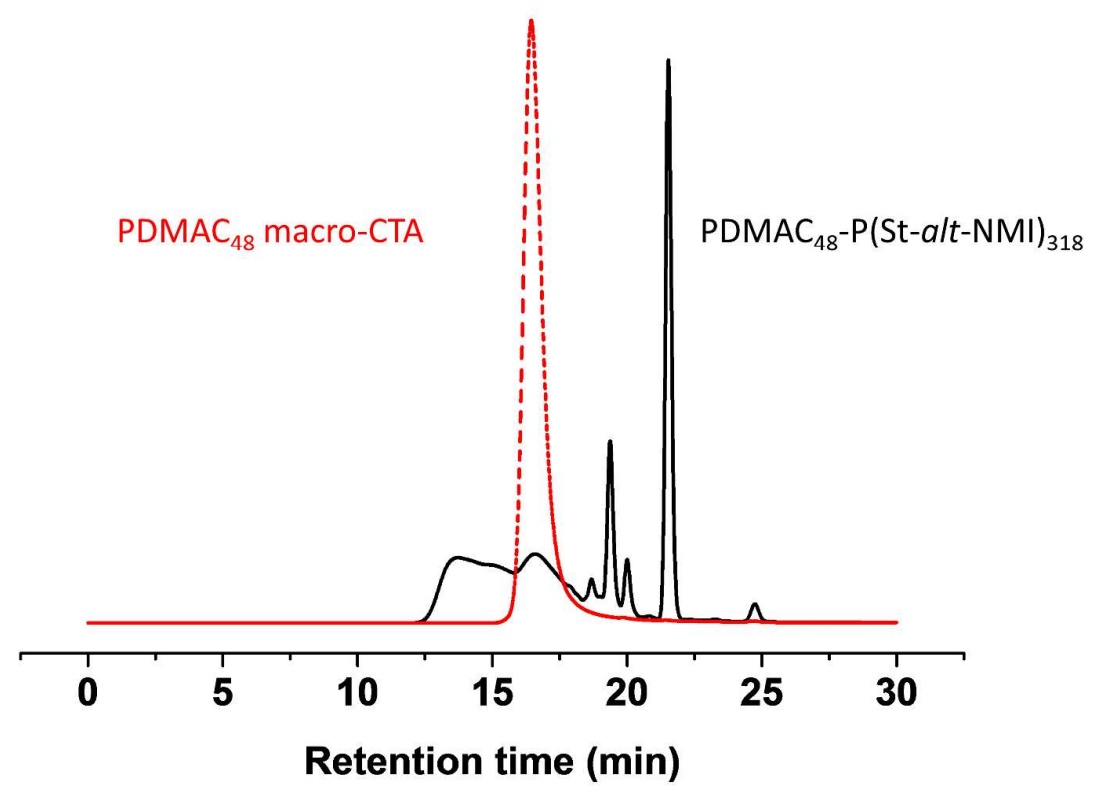

Figure S5. DMF GPC traces (UV detector) obtained for the PDMAC 48 macro-CTA, and the corresponding $\mathrm{PDMAC}_{48} \mathrm{P}(\mathrm{St}-\text { alt-NMI })_{318}$ diblock copolymer synthesized via RAFT dispersion alternating copolymerization in a 50/50 \% w/w ethanol/MEK mixture at $70{ }^{\circ} \mathrm{C}$. After chain extension, unreacted macro-CTA can be detected at a UV wavelength of $300 \mathrm{~nm}$, which corresponds to the RAFT end-group.

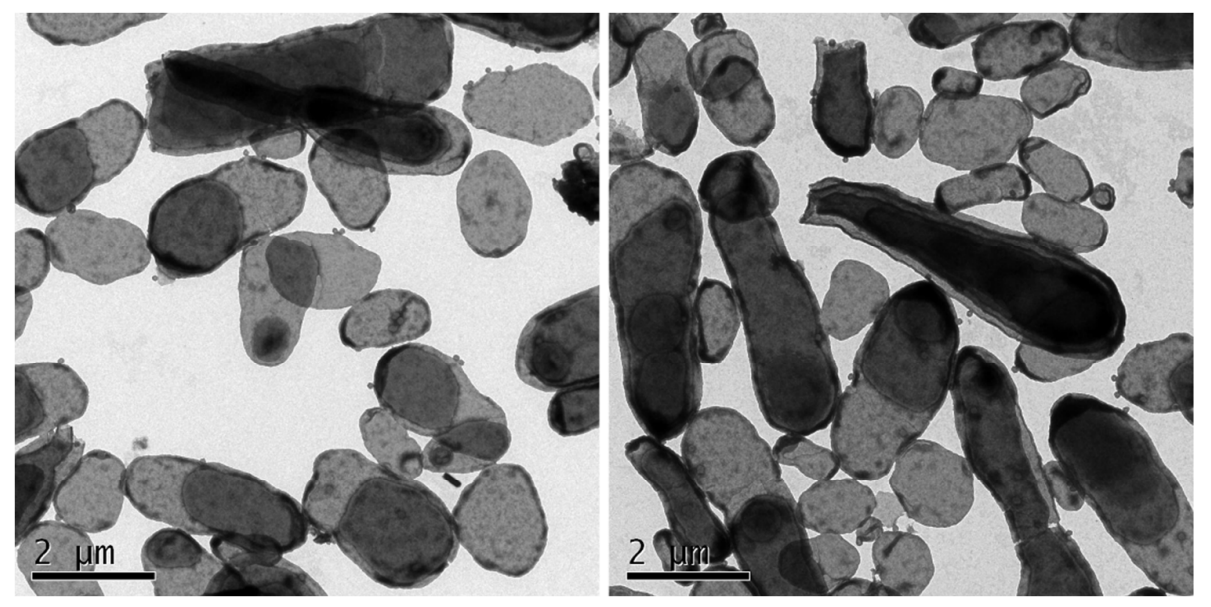

Figure S6. Additional TEM images recorded for $\mathrm{PDMAC}_{48} \mathrm{P}(\mathrm{St}-\text { alt-NMI })_{332}$ diblock copolymer oligolamellar vesicles (see entry 5 in Table 1) synthesized via RAFT dispersion polymerization in a 50/50 \% w/w ethanol/MEK mixture at $70{ }^{\circ} \mathrm{C}$. Conditions: Target diblock copolymer composition was $\mathrm{PDMAC}_{48}-\mathrm{P}(\mathrm{St}-\text { alt-NMI })_{350}$; $[\mathrm{St}] /[\mathrm{NMI}]$ feed molar ratio = 1.0; $[$ macro-CTA] $/[\mathrm{AIBN}]$ molar ratio $=10.0$; total solids concentration $=20 \% \mathrm{w} / \mathrm{w}$. 


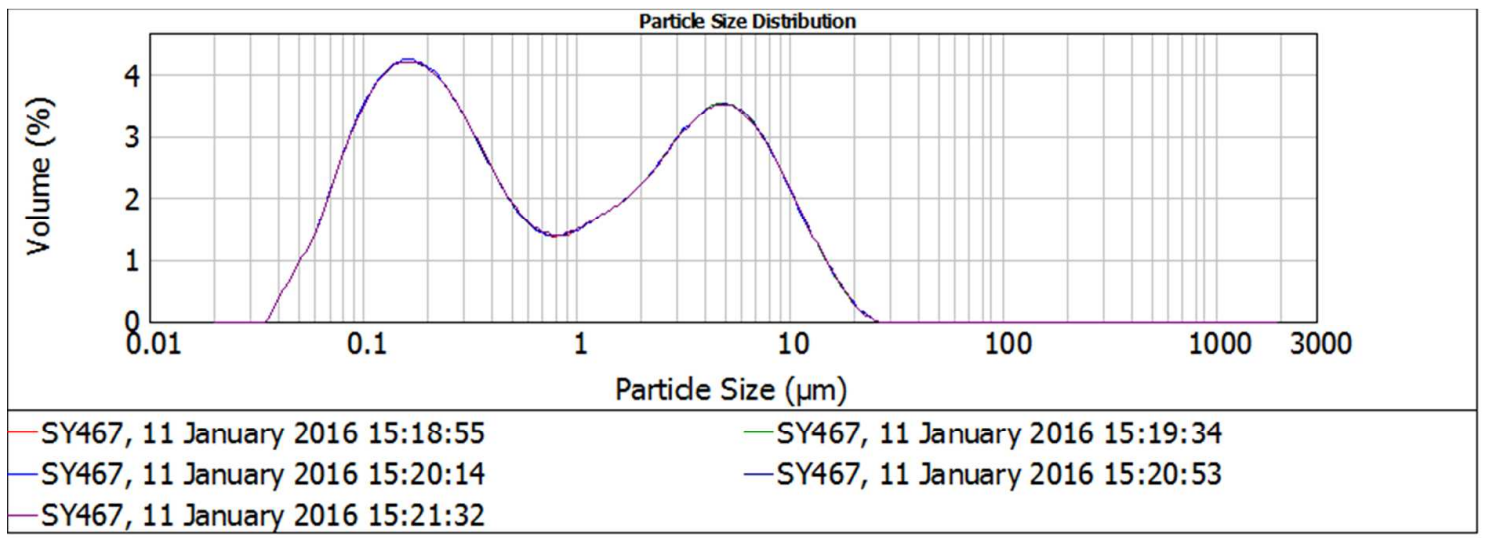

Figure S7. Volume-average particle diameter of $\mathrm{PDMAC}_{48}-\mathrm{P}(\mathrm{St}-\text { alt-NMI })_{332}$ diblock copolymer oligolamellar vesicles (see entry 5 in Table 1), as determined by laser diffraction.

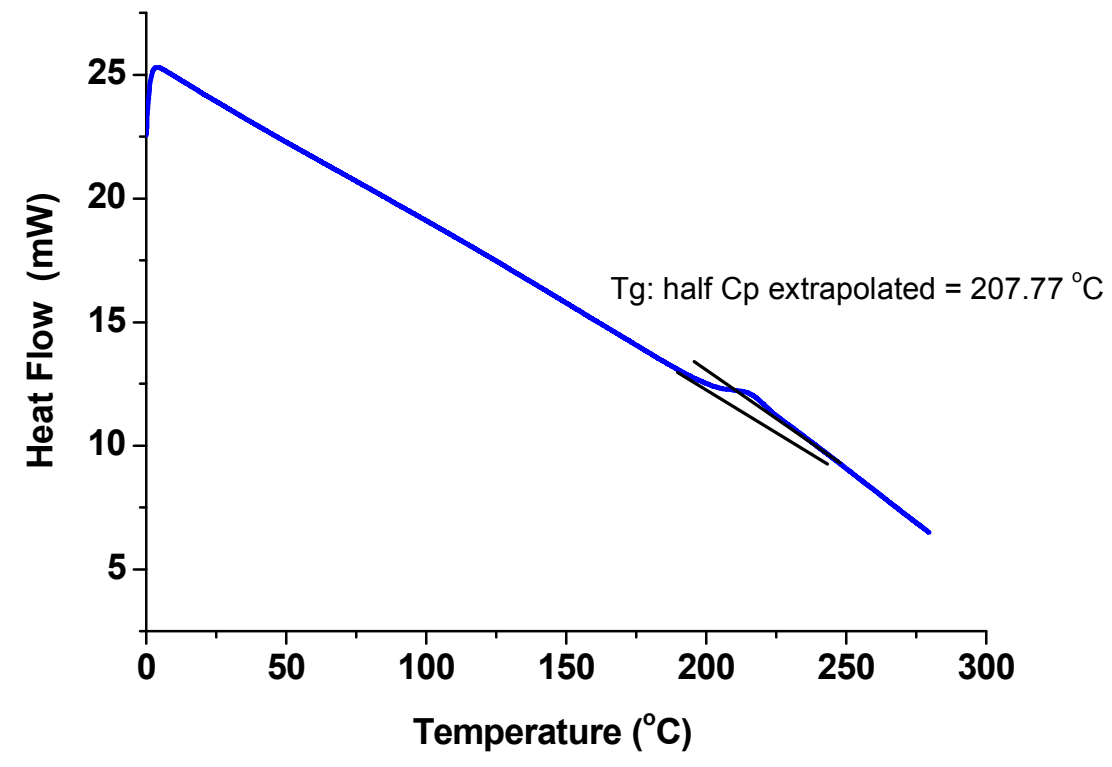

Figure S8. DSC curve obtained for $\mathrm{PDMAC}_{48}-\mathrm{P}(\mathrm{St}-\text { alt-NMI })_{332}$ diblock copolymer oligolamellar vesicles (see entry 5 in Table 1). Experiments were performed under a nitrogen atmosphere between $0{ }^{\circ} \mathrm{C}$ and $280{ }^{\circ} \mathrm{C}$ at a heating rate of $20{ }^{\circ} \mathrm{C} \mathrm{min}{ }^{-1}$. 

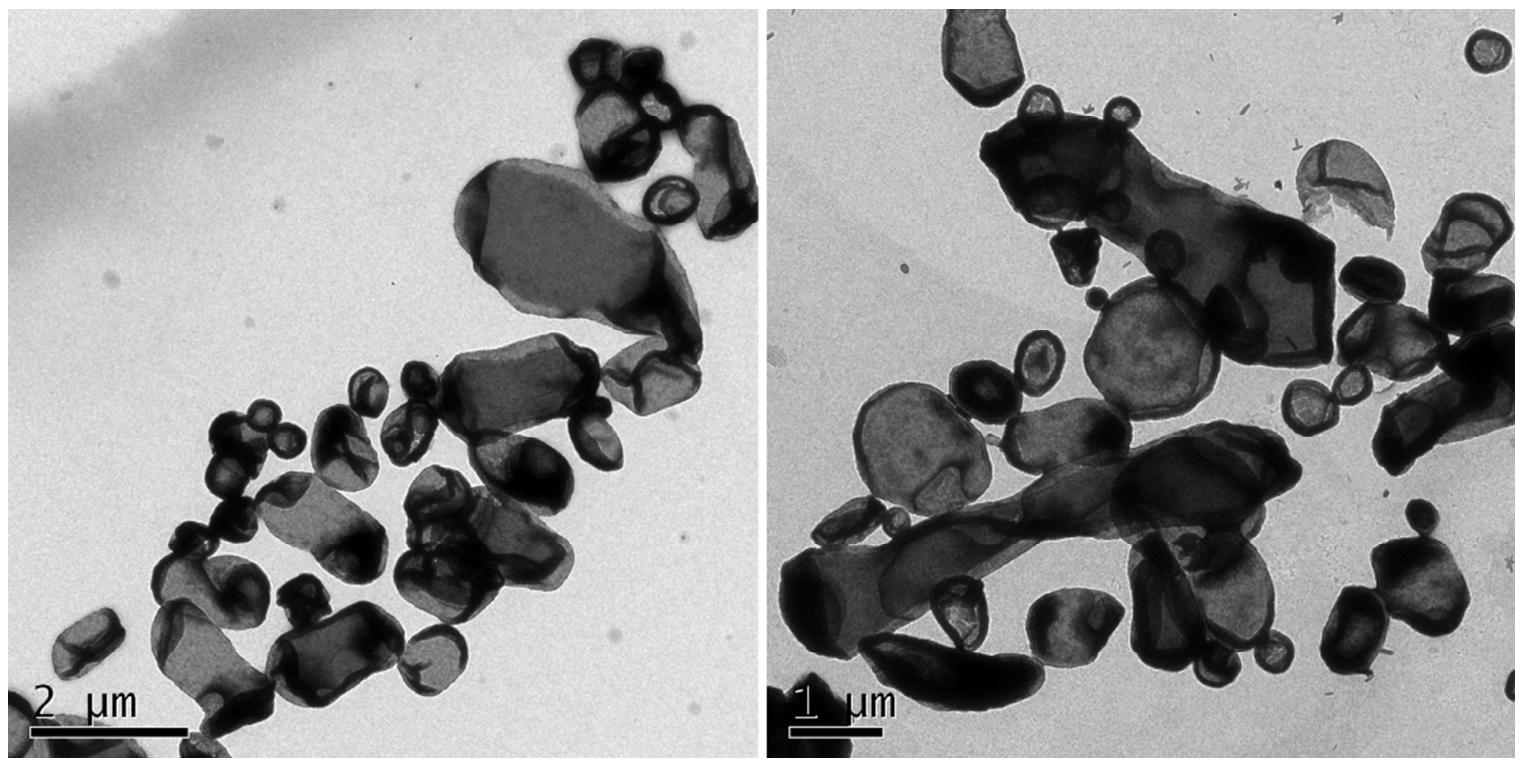

Figure S9. TEM images recorded for $\mathrm{PDMAC}_{48}-\mathrm{P}\left(\mathrm{St}-\right.$ alt-NMI) ${ }_{330}$ diblock copolymer (see entry 6 in Table 1) synthesized via RAFT dispersion alternating copolymerization of styrene with $\mathrm{N}$-phenylmaleimide in a 50/50 w/w ethanol/MEK mixture at $80{ }^{\circ} \mathrm{C}$ for $5 \mathrm{~h}$. Conditions: Target diblock copolymer composition was $\mathrm{PDMAC}_{48}-\mathrm{P}(\mathrm{St}-\text { alt-NMI)})_{350}$; [St]/[NMI] feed molar ratio $=1.0 ;[$ macro-CTA $] /[\mathrm{AIBN}]$ molar ratio $=10 ;$ copolymer concentration $=20 \%$ w/w solids. 


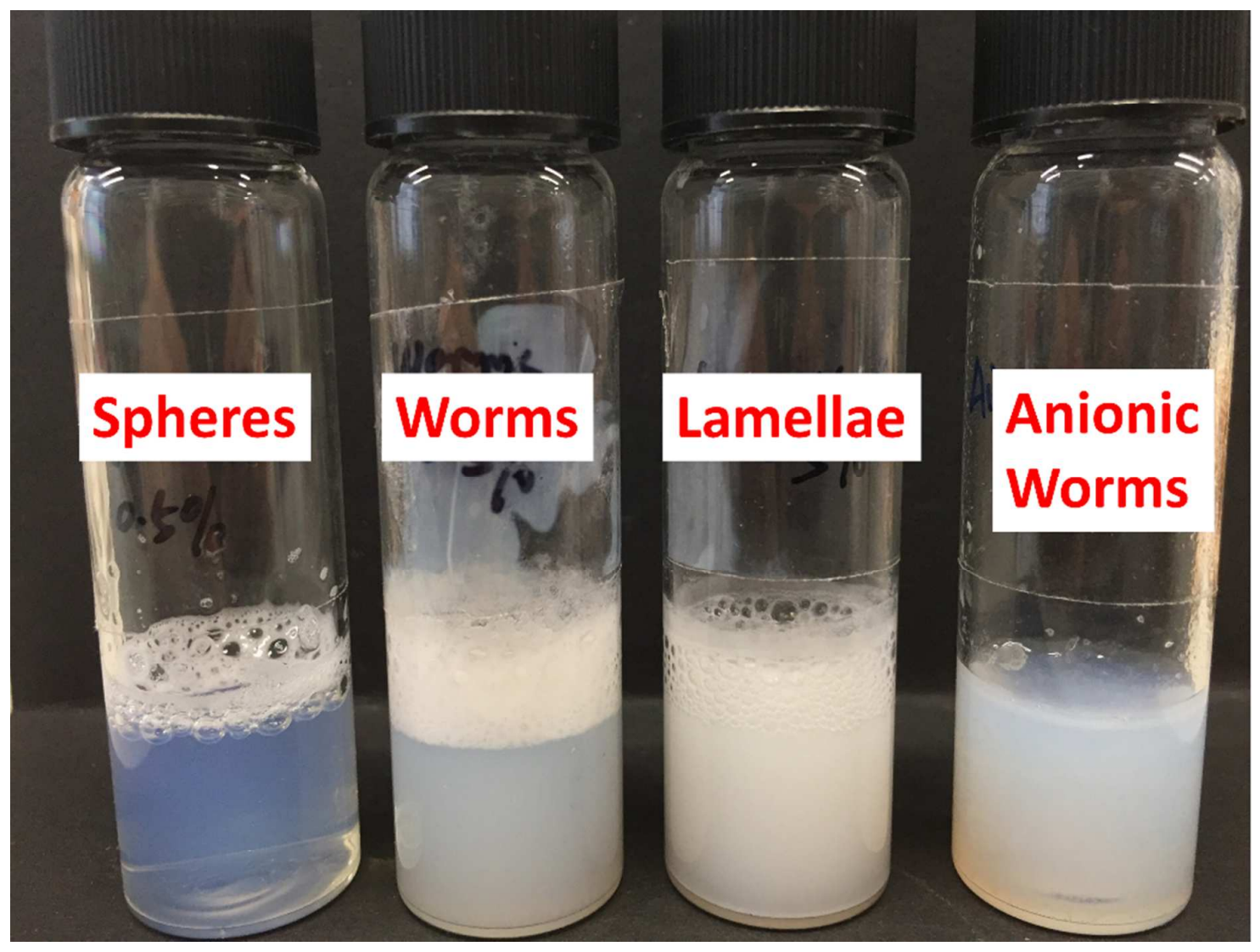

Figure S10. Digital photograph showing a series of four aqueous foams stabilized by $0.50 \%$ w/w PDMAC 48 -P(St-alt-NMI) $)_{\mathrm{x}}$ diblock copolymer nano-objects (spheres, $\mathrm{x}=142$; worms, $\mathrm{x}$ $=230$; oligolamellar vesicles, $\mathrm{x}=332$ ) recorded after homogenization and allowing the foams to stand for $30 \mathrm{~min}$ at $20{ }^{\circ} \mathrm{C}$, plus a control experiment conducted using $0.50 \% \mathrm{w} / \mathrm{w}$ anionic $\mathrm{PMAA}_{84} \mathrm{P}(\mathrm{St}-\text { alt-NMI })_{400}$ worms. The latter sample has an anionic stabilizer block, which prevents efficient adsorption at the anionic air/water interface. 